\title{
EVALUATION OF OPTICAL COHERENCE TOMOGRAPHY ANGIOGRAPHY IN DIABETIC RETINOPATHY
}

\author{
By
Maged Ali Boltia, Abd El-Moez Haddad Ahmed, and Abd El-Ghany Ibrahim Abd El-Ghany

Department of Ophthalmology, Faculty of Medicine, Al - Azhar University (Cairo)

Corresponding author: Maged Ali Boltia,

Mobile: 01110656246, E-mail: magedboltia@gmail.com

\begin{abstract}
Background: Diabetic retinopathy (DR) is a microvascular complication of diabetes, and it is a leading cause of blindness. Fluorescein angiography (FA) is a vitally important diagnostic tool for evaluating fundus features of DR.

Objective: To evaluate optical coherence tomography (OCT) angiography in eyes with diabetic retinopathy in comparison to findings in fundus fluorescein angiography.

Patients and Methods: This was a cross-sectional observational study conducted in the Ophthalmology Department of Sayed Galal Hospital from December 2016 to June 2017. Twenty eyes were included in the study with diabetic retinopathy with age between thirty three and sixty years of both genders, and those met the inclusion criteria. Patients were fully evaluated including history, examination and investigations. Investigations used Optical Coherence Tomography Angiography and Fundus Flouresien Angiography (FFA).
\end{abstract}

Results: The comparison between groups regarding qualitative data was done by using Chi-square test and/or Fisher exact test the patients ages mean value was $48.95 \pm 6.75$ years, of which $75 \%$ were females, and $25 \%$ were males. Microaneurysm was detected in all eyes in OCTA and FFA, retinal hemorrhage in DCP in $45 \%$ (9/20) of eyes compared to 15\% (3) in SCP with a significant difference that may allow better evaluation of level of retinal hemorrhage among diabetic eyes, detection of retinal capillary dropout. Areas of capillary loss obscured by fluorescein leakage on fluorescein angiography were more clearly defined on OCTA.

Conclusion: Ability of FFA and OCTA in microanerysms detection in all eyes, but less numerous in OCTA than FFA, and OCTA could precisely locate their depth and origin which is not possible in FFA. The assessment of capillary non-perfusion areas in our study by OCTA showed higher accuracy compared to FA but with a difference that was not statistically significant.

Keywords: Diabetic Retinopathy, Fluorescein Angiography, Optical Coherence Tomography Angiography.

\section{INTRODUCTION}

Diabetic retinopathy (DR) is a microvascular complication of diabetes mellitus and it is a leading cause of blindness (Nentwich and Ulbing, 2015). Because the number of patients with DR and vision-threatening DR is expected to increase. Further research is warranted on the methods for evaluating clinical conditions of DR and treatment advances (Bourne et al., 2013). Fluorescein angiography (FA) is a vitally important diagnostic tool for evaluating clinical 
fundus features of DR (Witmer et al., 2013). FA can detect primary vascular lesions as microaneurysms, and advanced vascular abnormalities such as venous beading and intraretinal microvascular abnormalities (IRMA). Retinal nonperfusion, which represents intraretinal capillary occlusion or dropout, can be visualized as a dark area surrounded by large retinal vessels. Neovascularization can be identified by remarkable leakage of the dye toward the vitreous cavity. Diagnosis of DR progression using FA could be integral to enabling decisions on treatment indications; however, intravenous dye injections should be performed carefully because patients with severe DR tend to have systemic vascular complications such as severe renal disorder and cardiovascular diseases (Kawasaki et al., 2011). Even in healthy subjects, dye injections can also occasionally cause nausea and, rarely but critically anaphylaxis (Kwiterovich et al., 2011).

Furthermore, FA cannot separately visualize the intraretinal structures of the major capillary networks; the images of the superficial and deep capillaries overlap because FA images are limited to two dimensions (Witmer et al., 2013).

Several theoretically-based OCT angiography methods were developed for three-dimensional non-invasive vascular mapping at microcirculation level (Spaide et al., 2015).

The present work aimed to evaluate optical coherence tomography (OCT) angiography in eyes with diabetic retinopathy in comparison to findings in fundus fluorescein angiography.

\section{PATIENTS AND METHODS}

This was a cross-sectional, observational study conducted in the Ophthalmology Department of Sayed Galal Hospital from December 2016 to June 2017.

Twenty eyes of twelve patients with diabetic retinopathy included in this study. Their ages ranged between thirty three and sixty years of both genders at outpatient clinic. All patients gave written consents to share in this work.

\section{Exclusion criteria:}

Dense media opacity interfering with imaging FFA images (as vitreous haemorrhage), macular edema due to conditions other than diabetic retinopathy, previous ocular surgeries.

All patients had standard clinical examination including: History taking including; personal data, history of systemic diseases particularly; hypertension, ocular diseases; mainly glaucoma, previous treatments; mainly retinal laser photocoagulation and/or intraocular injections). BCVA (using Snellen's visual acuity chart). Pupillary light reflex. IOP measurement (using Goldmann applanation tonometer). Slit lamp examination of the anterior segment. Dilated fundus examination (using slit lamp biomicroscopy with + 90D lens) Urea and Creatinine levels were done to evaluate kidney functions as fluorescein is excreted by the kidney. Multimodal imaging procedures including: Color fundus photography. Fluorescein angiography. Optical coherence tomography angiography. All the above 
tests were performed on the same day or within a week before imaging.

Fundus photography and FA: Both Fundus coloured photography and FFA were done after pupillary dilatation and intravenous injection of $5 \mathrm{ml}$ of $10 \%$ fluorescein sodium, using (DRI OCT Triton plus, Topcon, Tokyo, Japan) machine.

\section{Optical coherence tomography} angiography: Angio-OCT was done for all patients using the Topcon OCT instrument (DRI OCT Triton plus, Topcon, Tokyo, Japan) machine.

The Parameters used in the machine: Triton swept-source OCT uses a wavelength of $1050 \mathrm{~nm}$ with a scan speed of 100000 A-scans per second. The instrument employs an active eye tracker that follows the eye movement, detects blinking and adjusts the scan position accordingly, thereby reducing motion artifact during OCTA imaging. Scan size for retina is $6 \times 6 \mathrm{~mm}$. The macular scans were automatically segmented by the OCTA software into four 'en face' OCT slabs; (1) Superficial retinal slab (SRL) from $2.6 \mu \mathrm{m}$ beneath the internal limiting membrane to the $15.6 \mu \mathrm{m}$ beneath the interface of the inner plexiform layer and inner nuclear layer (IPL/INL), (2) Deep retinal slab (DRL) from the $15.6 \mu \mathrm{m}$ beneath the IPL/INL to $70.2 \mu \mathrm{m}$ beneath the IPL/ INL, (3) Outer retinal slab from $70.2 \mu \mathrm{m}$ beneath the IPL/INL to the Bruch's membrane (BM) and (4) Choriocapillaris from the BM to $10.4 \mu \mathrm{m}$ beneath the BM.

Image analysis of Fundus Examination for diffuse macular edema (DME), cystoid macular edema (CME), clinically significant macular edema
(CSME), non-proliferative diabetic retinopathy (mild, moderate or severe), and proliferative diabetic retinopathy.

FFA images were examined early, intermediate and late-phases of FFA images; central and peripheral images.

The following findings were evaluated on FFA images: Perifoveal capillary arcade (PFCA) of the capillary plexus, whether it was visible or not; disrupted or not. Central and Peripheral non perfused areas (Areas of capillary dropouts) in early phases. Dilated capillaries (Telangiectatic vessels) with staining of its walls in late-phases, areas of intraretinal hemorrhages. Areas of hyperfluorescence in the macula denoting diffuse or cystoid (petaloid) macular edema, in late phases, microaneurysms (MA). Disc hyperfluorescence (Disc leakage) in late phases. Photocoagulation scars. Neovascularization elsewhere (NVE) and disc neovascularization (NVD).

OCTA images: Angiographic analysis was done, focusing on the two vascular layers of the retina; SCP and DCP. The average capillary density, central capillary density and Foveal avascular zone (FAZ).

The following findings were evaluated at the level of both plexuses: FAZ interruption. Capillary non-perfusion areas. The presence of perifoveal changes (telangiectasia, dilatation or rarefaction). Haemorrhages. Macular edema, with further evaluation on the co-registered OCT en fac intensity images and OCT Bscan images. Microaneurysms (MA). Intraretinal Microvascular Abnormalities (IRMA). 
Quantitative analysis: Vessel density measurement was performed on the selected images; average and foveal capillary density were measured automatically by the instrument. Vascular density was calculated by the device as the percentage of the area occupied by blood vessels with flow above a threshold detection level in a selected area and depth of vessels .The FAZ in the SCP was manually outlined by the investigator and the FAZ area was calculated using the Triton software.

Qualititative analysis: Diabetic changes were also detected as microaneurysms, capillary non-perfusion, FAZ interruption, perifoveal changes, haemorrahges and edema in both Superficial and deep capillary plexuses.

\section{Statistical Analysis:}

Data were collected, revised, coded and entered to the Statistical Package for the Social Sciences (IBM SPSS) version 23. The quantitative data were presented as mean, standard deviations and ranges. Qualitative variables were presented as number and percentages. The comparison between groups regarding qualitative data was done by using Chi-square test and/or Fisher exact test when the expected count in any cell found less than 5. The confidence interval was set to $95 \%$ and the margin of error accepted was set to $5 \%$. P-value $<0.05$ was significant

\section{RESULTS}

The patients' ages ranged from thirty three to sixty years with mean value 48.95 \pm 6.75 years, of which $75 \%$ of eyes belonged to females, and $25 \%$ of eyes belonged to males. Diabetes mellitus duration ranged between six and sixteen years with mean $10.5 \pm 3.69$ years. $33 \%$ of patients were on insulin, $67 \%$ were on oral treatment among the patients with diabetes, and $25 \%$ had concomitant hypertension (Table 1).

Table (1): Demographic data of the studied cases (12cases)

\begin{tabular}{|l|l|c|}
\hline \multirow{2}{*}{ Age (years) } & Mean \pm SD & $48.95 \pm 6.75$ \\
\cline { 2 - 3 } Gender & Range & $33-60$ \\
\cline { 2 - 3 } & Females & $9(75.0 \%)$ \\
\cline { 2 - 3 } Duration of DM (years) & Males & $3(25.0 \%)$ \\
\cline { 2 - 3 } & Mean \pm SD & $10.55 \pm 3.69$ \\
\cline { 2 - 3 } Type of treatment & Range & $6-16$ \\
\hline \multirow{2}{*}{ Concomitant disease } & Oral drugs & $8(67.0 \%)$ \\
\cline { 2 - 3 } & Insulin & $4(33.0 \%)$ \\
\cline { 2 - 3 } & Non & $9(75.0 \%)$ \\
\cline { 2 - 3 } & HTN & $3(25.0 \%)$ \\
\hline
\end{tabular}

FFA was performed for all the patients and revealed $100 \%$ of eyes had micro aneurysms. New vessels were found in $40 \%$ of eyes, IRMA/collaterals were found in $35 \%$ of eyes, paracentral capillary dropout was found in $60 \%$ of eyes. DME was found in $45 \%$ of eyes, while CME was found in $20 \%$ of eyes. Also, CSME was found in $20 \%$ of eyes and central DMI in $60 \%$ of eyes. $15 \%$ of 
eyes had mild NPDR, $40 \%$ of eyes had moderate NPDR, $5 \%$ of eyes had severe
NPDR and, and $40 \%$ of eyes had PDR (Table 2).

Table (2): Descriptive data found by Fundus Fluorescein Angiography (FFA) in studied cases

\begin{tabular}{|l|l|c|c|}
\hline \multicolumn{2}{|c|}{ FFA } & No. & \% \\
\hline Microaneurysms & Yes & 20 & $100.0 \%$ \\
\hline New vessels & Yes & 8 & $40.0 \%$ \\
\hline IRMA/collaterals & Yes & 7 & $35.0 \%$ \\
\hline Paracentral cap dropout & Yes & 12 & $60.0 \%$ \\
\hline \multirow{4}{*}{ Macular oedema } & DME & 9 & $45.0 \%$ \\
\cline { 2 - 4 } & CME & 4 & $20.0 \%$ \\
& CSME & 4 & $20.0 \%$ \\
\cline { 2 - 4 } & Negative & 3 & $15.0 \%$ \\
\hline \multirow{4}{*}{ Retinopathy staging } & Mild NPDR & 3 & $15.0 \%$ \\
\cline { 2 - 4 } & Moderate NPDR & 8 & $40.0 \%$ \\
\cline { 2 - 4 } & Severe NPDR & 1 & $5.0 \%$ \\
\cline { 2 - 4 } & PDR & 8 & $40.0 \%$ \\
\hline
\end{tabular}

$\mathrm{FFA}=$ Fundus fluorescein angiography, DME= Diffuse Macular Edema, CME= Cystoid Macular Edema, $\mathrm{CSME}=$ clinically significant macular edema, DMI= Diabetic Macular Ischaemia, NPDR= Non-Proliferative Diabetic Maculaopathy, PDR= Proliferative Diabetic Maculopathy.

The average Capillary density (AvCD) ranged 37.51- 52.2\% with a mean of $43.32 \pm 3.82 \%$, while the central capillary density (Central CD) ranged 17.28-37.93
$\%$ with mean $27.74 \pm 7.14 \%$, and the FAZ area ranged between $0.2-1.07 \mathrm{~mm}^{2}$ with mean $0.46 \pm 0.27 \mathrm{~mm}^{2}$ (Table 3).

Table (3): Descriptive data found by OCTA in the studied eyes

\begin{tabular}{|l|l|c|}
\hline \multicolumn{2}{|c|}{ OCTA overview } & No. $=\mathbf{2 0}$ \\
\hline \multirow{2}{*}{ Av CD } & Mean \pm SD & $43.32 \pm 3.82 \%$ \\
\cline { 2 - 3 } & Range & $37.51-52.2 \%$ \\
\hline \multirow{2}{*}{ Central CD } & Mean \pm SD & $26.07 \pm 5.72 \%$ \\
\cline { 2 - 3 } & Range & $17.28-37.93 \%$ \\
\hline \multirow{2}{*}{ FAZ area $\left(\mathrm{mm}^{2}\right)$} & Mean $\pm S D$ & $0.46 \pm 0.27 \mathrm{~mm}^{2}$ \\
\cline { 2 - 3 } & Range & $0.2-1.07 \mathrm{~mm}^{2}$ \\
\hline
\end{tabular}

OCTA= Optical Coherence Tomography Angiography, AV CD= Average Capillary Density, $\mathrm{CD}=$ Capillary Density, $\mathrm{SD}=$ Standard Deviation, $\mathrm{FAZ}=$ Foveal Avascular Zone

Comparison between results by OCTA SCP and DCP of the studied eyes, OCTA showed pathological diabetic changes in 2 regions SCP and DCP. FAZ interruption was detected in SCP in $40 \%$ of the eyes compared to $50 \%$ in DCP with $p$ value $=0.789$ which was not statistically significant. $45 \%$ of eyes were distorted in the SCP imaging compared to $35 \%$ in DCP imaging, Capillary non- perfusion could be detected in SCP imaging in 80 of eyes compared to $70 \%$ in DCP imaging with $\mathrm{p}$ value $=0.465$ which was not statically significant, perifoveal changes, in SCP imaging, rarefaction was seen in $5 \%$ of eyes, dilatation in $35 \%$, rarefaction and dilatation in $40 \%$. In DCP imaging, the results were $20 \%, 40 \%$ and $10 \%$ of the eyes respectively where all of no statistically significant difference. 
Haemorrhages were detected only in $15 \%$ of eyes in SCP while $45 \%$ of the eyes showed haemorrhages in DCP imaging with $\mathrm{p}$ value $=0.038$ which was a statistically significant difference. Regarding edema, there was no statistically significant difference with $p$ value $=1.000$ as SCP showed $40 \%$ of eyes with edema and DCP showed $40 \%$ of eyes, and microaneurysms were easily shown by SCP in $100 \%$ of eyes, and $100 \%$ of eyes by DCP with $\mathrm{p}$ value $=0.315$ which was statistically not applicable (Table 4).

Table (4):Comparison between OCTA SCP and DCP abnormalities

\begin{tabular}{|l|l|c|c|c|c|c|}
\hline \multirow{2}{*}{ Groups } & \multicolumn{2}{c}{ SCP } & \multicolumn{2}{c|}{ DCP } & \multirow{2}{*}{ P-value } \\
\cline { 3 - 7 } \multicolumn{2}{|c|}{ Parameters } & No. & \% & No. & \% & \\
\hline \multirow{2}{*}{ FAZ interruption } & Yes & 8 & $40.0 \%$ & 10 & $50.0 \%$ & \multirow{2}{*}{0.492} \\
\cline { 2 - 7 } & Distortion & 9 & $45.0 \%$ & 7 & $35.0 \%$ & \\
\hline Cap non perf & Yes & 16 & $80.0 \%$ & 14 & $70.0 \%$ & 0.465 \\
\hline \multirow{3}{*}{ Perifoveal changes } & Dilatation & 7 & $35.0 \%$ & 8 & $40.0 \%$ & \multirow{2}{*}{0.118} \\
\cline { 2 - 6 } & Rarefaction & 1 & $5.0 \%$ & 4 & $20.0 \%$ & \\
\cline { 2 - 6 } & $\begin{array}{l}\text { Rarefaction, } \\
\text { dilatation }\end{array}$ & 8 & $40.0 \%$ & 2 & $10.0 \%$ & \\
\hline Hemorrhages & Yes & 3 & $15.0 \%$ & 9 & $45.0 \%$ & 0.038 \\
\hline Edema & Yes & 7 & $35.0 \%$ & 8 & $40.0 \%$ & 0.743 \\
\hline Microaneurysms & Yes & 20 & $100.0 \%$ & 20 & $100.0 \%$ & 1 \\
\hline IRMA & Yes & 8 & $40.0 \%$ & 5 & $25.0 \%$ & 0.311 \\
\hline
\end{tabular}

*:Chi-square test

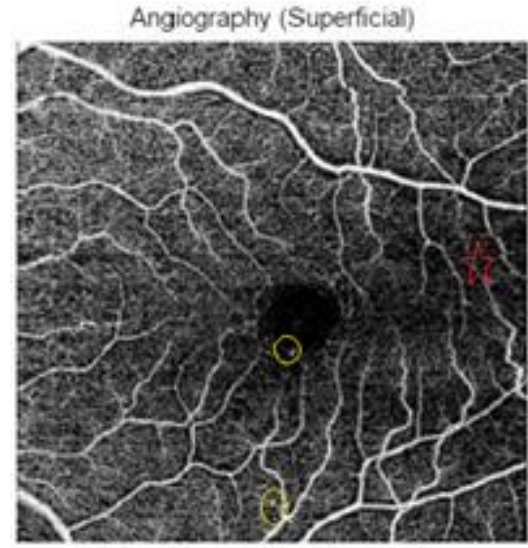

$\mathrm{ILM}+2.6 \mu \mathrm{m} \sim \mathrm{IPLINL}+156 \mu \mathrm{m}$

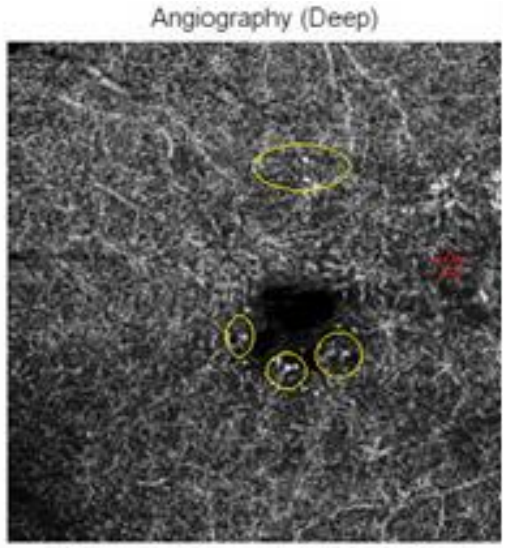

IPUINL + $15.6 \mu \mathrm{m} \sim$ IPUINL + $70.2 \mathrm{\mu m}$

Figure (1): OCTA SCP (LT pic) and DCP (RTpic) of OD eye of a 52 years old female diabetic patient for 8 years showed microaneurysms (surrounded by yellow circles) more numerous in DCP, enlaged and interrupted FAZ in DCP.

Both FFA and OCTA detected the presence of microaneurysms in all eyes of the study.

FFA detected IRMAICollaterals in $35 \%$ of eyes, while OCTA detected $40 \%$ of eyes with $p$ value $=0.743$ which was not significant. Similarly capillary nonperfuson showed no statistically significant difference between FFA and OCTA as FFA detected in $60 \%$ of eyes, and OCTA detected in $80 \%$ of eyes with $\mathrm{p}$ value $=0.243$ (Table 5) . 
Table (5): Diabetic pathological changes detected in FFA in comparison with OCTA of SCP

\begin{tabular}{|c|c|c|c|c|c|}
\hline \multirow{2}{*}{ Groups } & \multicolumn{2}{|c|}{ SCP } & \multicolumn{2}{c|}{ FFA } & \multirow{2}{*}{$\begin{array}{c}\text { P- } \\
\text { value }\end{array}$} \\
\cline { 2 - 5 } & No. & $\%$ & No. & \% & 1 \\
\hline Microaneurysms & 20 & $100.0 \%$ & 20 & $100.0 \%$ & 1 \\
\hline IRMA & 8 & $40.0 \%$ & 7 & $35.0 \%$ & 0.744 \\
\hline Non perfusion & 16 & $80 \%$ & 12 & $60 \%$ & 0.168 \\
\hline
\end{tabular}

*:Chi-square test

Overall, diabetic macular edema could be detected in $17(85 \%)$ eyes by FFA, while only in $35 \%$ of eyes by OCTA of SCP, and in $40 \%$ of eyes by OCTA of DCP. Thus, a significant difference was found between SCP and FFA. The same statistically significant difference was found in DCP compared to FFA. However, there was no significant difference between SCP and DCP was found (Table 6).

Table (6): Overall prevalence of macular edema among all cases by clinical examination, FFA and OCTA examination of SCP and DCP

\begin{tabular}{|c|c|c|c|c|}
\hline \multicolumn{2}{|c|}{ Edema } & No. & \% & P-value \\
\hline \multirow{3}{*}{$\begin{array}{c}\text { FFA } \\
17(85 \%)\end{array}$} & clinical & 7 & $35.0 \%$ & $<0.002$ \\
\cline { 2 - 5 } & $\begin{array}{c}\text { OCTA } \\
\text { SCP }\end{array}$ & 7 & $35 \%$ & $<0.002$ \\
\cline { 2 - 5 } & $\begin{array}{c}\text { OCTA } \\
\text { DCP }\end{array}$ & 8 & $40 \%$ & $<0.004$ \\
\hline
\end{tabular}



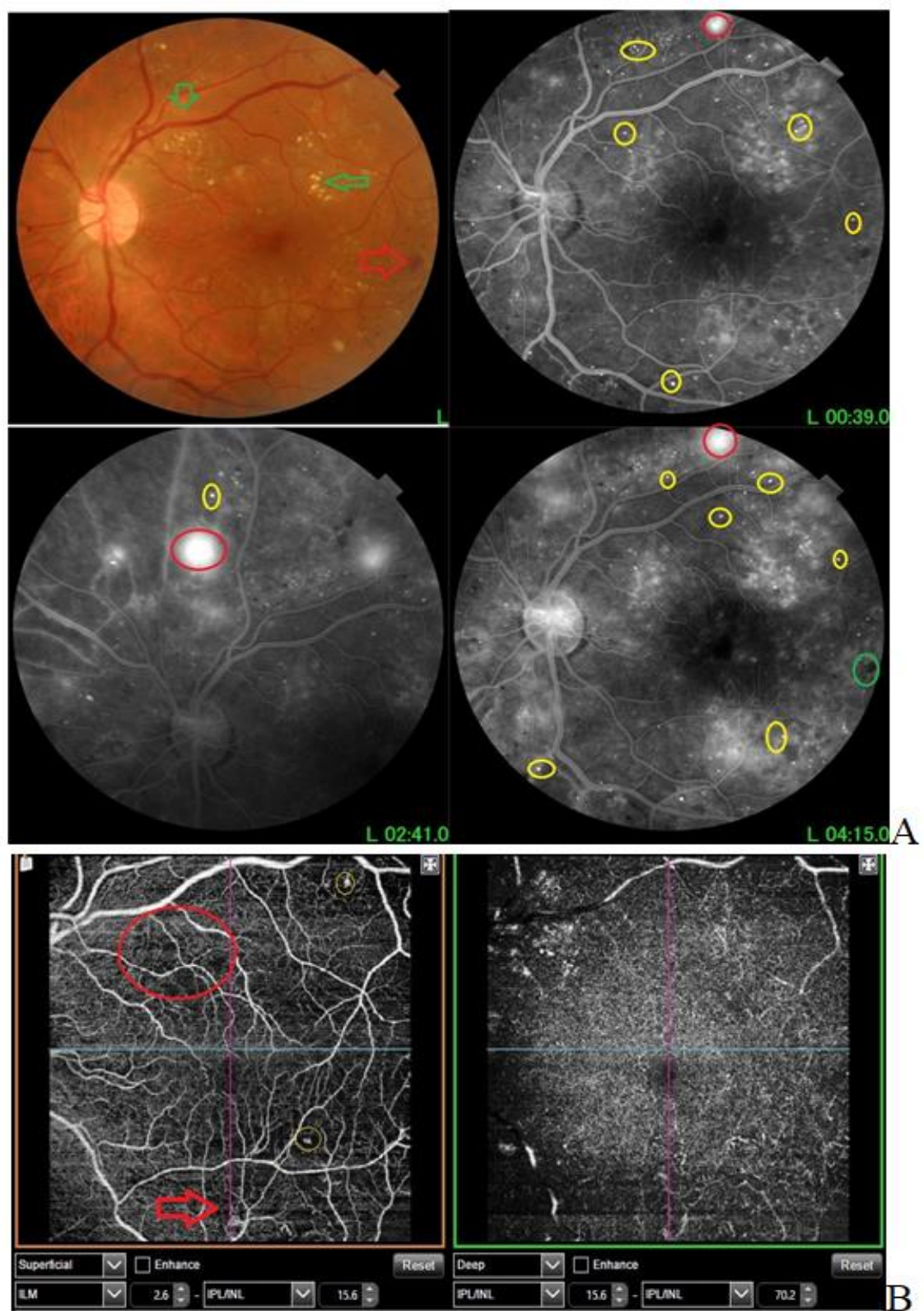

Figure (3): (eye no. 2): (A) :Colored fundus photo and FFA of LT eye of 42 years old male patient with 8 years duration of diabetes:colored Fundus photo shows hard exudates(pointed by green arrows), haemorrhage (pointed by red arrow), FFA shows leaking and non leaking microaneurysms causing macular edema(examples surrounded by yellow circle)more numerous than OCTA, evidence of blocked fluroescence corresponding to hemorrhage seen in colored photo (examples surrounded by green circle), evidence of NVEs(examples surrounded by red circle)(not appear in OCTA due to narrower field), NVD and perifovea capillary drop out.

(B): OCTA of LT eye of the same patient, SCP and DCP showings: microaneurysms(examples surrounded by yellow circle) less numerous than FFA due to narrower field and low blood flow in them, capillary non perfusion areas(examples surrounded by red circle) that appear more clearly than FFA and IRMA pointed by red arrow not appear in FFA. 


\section{DISCUSSION}

In this study OCTA allowed visualization of microaneurysms which were seen as focally dilated and abnormally shaped capillaries detected in both layers and detected in all eyes in FFA and OCTA but less numerous in OCTA than FFA due to narrower field and low blood flow in them in .OCTA could precisely locate their depth and origin as well which is not possible in FFA.

Salz et al. (2016) found that OCTA did have the added benefit of localizing the exact intraretinal depth of microaneurysms. Study presented by Ishibazawa et al. (2015) in patients with diabetes, declared more numerous microaneurysms shown on FA and those on OCTA, and they referred this to the histopathological configuration of microanerysms lumen that consists of diverse components such as thickened, hyalinized, fibrous, laminated, and lipidcontaining basement membrane, as well as hypercellular or multilayered endothelial cells that causes turbelance in the blood flow inside them and thus they suggested that the blood flow inside some types of microaneurysms may not have been perfectly displayed using OCTA, also they stated that around $80 \%$ of microaneurysms were seen to be located in the inner deep plexus.

Similarly, Salz et al. (2016) stated that OCTA could not detect all microaneurysms detected by FFA. They suggested that focal staining of vessel walls and the dye collection in the abnormally dilated blood vessel cause them appear brightly hyperfluorescent compared with the surrounding retina; also some microaneurysms can be seen to be leaking over time, which highlights and exaggerates their appearance, while OCTA relies on erythrocyte movement and the images are acquired over a very short time. Thus, the OCTA segmented images do not show leakage, which is why microaneurysms may seem smaller and less prominent on OCTA than FA that may allow better identification of microanerysm in the fluorescein angiography images, compared to OCTA.

Histopathologic studies on microaneurysms have described several kinds of microanuerysms, including those with high flow, those with minimal blood flow, and some that are completely occluded and may not have any flow. Therefore, OCTA may preferentially detect microaneurysms with higher flow and have a low sensitivity for microaneurysms with lower flow ( $\mathrm{Salz}$ et al., 2016).

OCTA has allowed us to visualize the morphology of IRMA using en face images. IRMAs are seen on OCTA as dilated or looping vessels near areas of capillary loss (Schaal et al., 2017). OCTA allows the detection of retinal neovascularization by observing flow signal above the internal limiting membrane (Carlo et al., 2016).

IRMA could be detected in our study by OCTA in more than FFA but with statistically insignificant difference and this was consistent with the result presented by Couturier et al. (2015) who revealed a very well ability of OCTA to detect them in all cases and referred this to the nature of IRMA that could have a more rapid blood flow than microaneurysms, thus may enhance their detection by OCTA. 
Salz et al. (2016) also found that OCTA did show vascular abnormalities that were not seen clinically or on FFA, although there was no ability to quantify these results. The reason for this could be, the fact that OCTA does not require dye means there is no confusion of the characteristics of the retina around the microaneurysm as occurs with hyperfluorescence from dye leakage on FA, so many of the microaneurysms and other vascular abnormalities were visualized to be in localized areas of nonperfusion, also the higher image resolution of the OCTA means that very small microvascular abnormalities that are visible on the OCTA may not be discernable on the FA owing to its lower resolution.

In this study, OCTA could visualize IRMA in SCP slightly more than in DCP with a non-statistically significant difference. Hwang et al. (2015) reported that IRMA was similarly identified with OCTA and FFA with no difference. On the other hand, Ishibazawa et al. (2015) stated better visualization of IRMA in deeper capillaries layer by OCTA than superficial one and referred this to that the thickness of the inner retina in the nonperfused area was thinner than that of normal parts of the retina.

The assessment of capillary nonperfusion areas in our study by OCTA showed slightly higher accuracy compared to FA but with a difference that was not statistically significant. They were visualized as capillary non visible areas between retinal vessels. Couturier et al. (2015) reported that edges of capillary non-perfusion areas are better delimited on OCTA than on FA, and some capillary non-perfusion areas detected on OCTA were visualized as perfused on FA. They referred this to superposition of the twocapillary plexuses and some early leakage on FA.

Ishibazawa et al. (2015) found that average area of retinal non-perfusion in the superficial plexus was slightly larger than that in the deep plexus but they clarified that there is no histopathologic evidence of the difference between the extent of retinal non-perfusion in superficial and deep plexuses and they suggested that these results might suggest that deep capillaries could to a certain extent be spared from microthrombosis compared with superficial ones.

Our study showed the enlargement and interruption of the FAZ by retinal capillary loss in areas of the macula (capillary dropout). According to recent studies, FAZ and parafoveal VD have been reported as good indicators of vascular dropout in DM patients (Sim et al., 2013).

OCTA could clearly identify capillary non-perfusion in DR eyes as well as it could distinguish the level of capillary drop out that may indicate ischemia between superficial and deep capillary plexuses, this identification of DCP by OCT angiography plays an important role in the setting of DM as experimental studies have shown that the DCP contributes to photoreceptor inner segment oxygen requirements (10-15\%), particularly during dark adaptation (Scarinci et al., 2016).

Our study could identify the perifoveal vascular changes including rarefaction, dilatation and telangiectasia by OCTA with delineation and distinguishment of 
level in superficial and deep capillary plexus. Salz et al. (2016) reported that OCTA allowed better identification of combined vascular changes in deep layer rather than superficial layer compared to FFA which was capable of a wider field of view, but an attempt to focus on the macula resulted in lower-resolution images, making it difficult to visualize small microvascular abnormalities. Couturier et al. (2015) concluded that SCP capillary rarefaction was better identified on OCT angiography.

Nevertheless, Couturier et al. (2015) stated that the DCP non-perfused areas were observed in only $35 \%$ of the subjects studied, while in our study they could be observed in $80 \%$ in SCP and $70 \%$ in DCP. In spite of that OCTA could detect in present study FAZ interruption in SCP in $40 \%$ and in DCP in 50\%. One of limitations in present study is that we did not evaluate the change in FAZ size over time in patients with diabetic macular edema.

FAZ area measured by OCTA ranged between $0.2-1.07 \mathrm{~mm}$ square. The FAZ area was well visualized on OCTA better than FFA and FAZ enlargement was detected in all the cases. This was in agreement with Kim et al. (2013) and AlSheikh et al. (2016).

OCTA allows evaluation of macular edema level where it was detected in images of the deep plexus in $40 \%$ of eyes and superficial plexus in $35 \%$ of eyes.

FFA could detect DME in $85 \%$ compared to overall edema detection in both DCP and SCP $75 \%$ by OCTA, and that may be referred to artifact in the OCTA technique, a decrease in flow velocity that may have fallen below the threshold level distinguishing low flow from noise on OCTA, fluid in DME may cause shadowing on OCTA and loss of OCT signal at deeper segmentations, or a combination of these factors .That was consistent with the result presented by Aditya et al. (2017) whose study used summated data and consecutive images with recording descriptive changes seen in retinal vasculature between visits on OCTA images of the superficial, deep and summated plexuses and suggested that summated images could allow better delineation.

The present study could detect retinal hemorrhage in DCP in $45 \%$ of eyes compared to $15 \%$ in SCP with a significant difference that may allow better evaluation of level of retinal hemorrhage among diabetic eyes. We compared OCTA and FA images, and evaluate relative sensitivity of OCTA compared to FFA which used to be the gold standard for evaluation of DME but with several limitations related to need of injection of dye. The data showed that OCTA visualized all of the microaneurysms visualized by FA. OCTA was able to appreciate the level of microaneurysms that was not detected by FA.

OCTA also successfully detected other abnormalities that were better evident compared to FA such as perifoveal microvascular changers including rarefaction, dilatation and telangiectasia. It also provides visualization of areas of capillary non-perfusion including areas too small to visualize on FA with ability of detection of diabetic macular edema and the level of accumulated fluid in addition to retinal hemorrhage level. 
There were limitations in our study related to the small sample size, small retinal area examined, patients with poor fixation led to lower images quality as high quality OCTA imaging requires patients to remain stable for several seconds during scanning in order to obtain high quality images, also some patients included were imaged by FFA and OCTA in the same day that could cause exhaustion, also automated segmentation software may fail in patients with edema when examining each plexus individually.

\section{CONCLUSION}

Ability of FFA and OCTA in microaneurysms detection in all eyes, but OCTA could precisely locate their depth and origin which is not possible in FFA. The assessment of capillary non-perfusion areas in our study by OCTA showed higher accuracy compared to FA but with a difference that was not statistically significant. Inability of OCT angiography to assess peripheral retinal involvement, hoping in the future that the advances in Swept-Source OCT angiography will provide us with machines that can perform imaging with faster scanning speed, thus yielding images with larger scanning areas and better resolution in a trial to overcome the limited field of view.

\section{REFERENCES}

1. Aditya G, Gill A, De Carlo T and Louzada RN (2017): Visualization of changes in the foveal avascular zone in both observed and treated diabetic macular edema using optical coherence tomography angiography. International Journal of Retina and Vitreous, 3: 1-11.

2. Al-Sheikh M, Akil H, Pfau M and Sadda SR (2016): Swept-Source OCT Angiography Imaging of the Foveal Avascular Zone and Macular Capillary Network Density in
Diabetic Retinopathy. Invest Ophthalmol Vis Sci., 57:3907-13.

3. Bourne RR, Rupert RA, Gretchen A , White RA, Smith J L, Flaxman SR, Price H and Pesudovs K (2013): Causes of vision loss worldwide, 1990-2010: a systematic analysis. The lancet Global Health, 1, (6); e339-e349.

4. Carlo TE, Salz DA, Waheed NK, Baumal and Duker (2016): Visualization of the Retinal Vasculature using Wide-Field Montage Optical Coherence Tomography Angiography. Ophthalmic Surg Lasers Imaging Retina, 46: 611-616.

5. Couturier A, Mane V, Bonnin S, Erginay A, Massin P, Gaudric A and Tadayoni R. (2015): Capillary plexus anomalies in diabetic retinopathy on optical coherence tomography angiography. Retina, 35:2384-2391.

6. Hwang TS, Jia Y, Gao SS, Bailey S, Lauer A, Flaxel C and Huang D (2015): Optical coherence tomography angiography features of diabetic retinopathy. Retina, 35(11):23712376.

7. Ishibazawa A, Nagaoka T, Takahashi A, Omae T, Tani T, Sogawa K and Yoshida A (2015): Optical Coherence Tomography Angiography in Diabetic Retinopathy: A Prospective Pilot Study. Am J Ophthalmol., 160(1):35-44.e1.

8. Kawasaki R, Cheung N, Islam FM, Klein R and Cotch $M$ (2011): Is diabetic retinopathy related to subclinical cardiovascular disease? Ophthalmology, 118(5):860-865.

9. Kim DY, Fingler J, Zawadzki RJ, Park S , Morse L, Schwartz and Dand Werner J (2013): Optical Imaging of the Chorio-retinal Vasculature in the Living Human Eye. Proc Natl Acad Sci USA, 110(35): 14354-59.

10. Kwiterovich KA, Maguire MG, Murphy RP, Schachat A, Bressler M, Bressler B and Fine L. (2011): Frequency of adverse systemic reactions after fluorescein angiography. Results of a prospective study. Ophthalmology, 98(7):1139-1142.

11. Nentwich MM and Ulbing MW (2015): Diabetic retinopathy - ocular complications of 
diabetes mellitus. World J Diabetes, 6: 489499.

12. Salz DA, de Carlo TE, Adhi M, Moult E, Choi W, Baumal $R$ and Waheed N (2016): Select features of diabetic retinopathy on swept-source optical coherence tomographic angiography compared with fluorescein angiography and normal eyes. JAMA Ophthalmol., 134(6):644-650.

13. Scarinci F, Nesper $P$ and Fawzi AA (2016): Deep retinal capillary non-perfusion is associated with photoreceptor disruption in diabetic macular ischemia. Am J Ophthalmol, 168:129-38.

14. Schaal KB, Munk MR, Wyssmueller I, Berger E, Zinkernagel S and Wolf S (2017): Vascular Abnormalities in Diabetic Retinopathy Assessed with Swept-Source
Optical Coherence Tomography Angiography Widefield Imaging. Retina, 39(1):79-87.

15. Sim DA, Keane P, Zarranz-Ventura J, Fung S, PownerB, Platteau $E$ and Egan A (2013): The effects of macular ischemia on visual acuity in diabetic retinopathy. Invest Ophthalmol Vis Sci., 54(3):2353-2360.

16. Spaide RF, Klancnik JM and Cooney MJ (2015): Retinal vascular layers imaged by fluorescein angiography and optical coherence tomography angiography.

JAMA Ophthalmol., 133(1):45-50.

17. Witmer MT, Parlitsis G, Patel S and Kiss S (2013): Comparison of ultra-widefield fluorescein angiography with the Heidelberg Spectralis ${ }^{\circledR}$ noncontact ultra-widefield module versus the Optos $^{\circledR} \quad$ Optomap $^{\circledR} \quad$ Clin Ophthalmol.,7 (13): 389-394. 
تقييم التصوير المقطحي الترابطي للأوعية الدموية في إعتلال الشبكية السكري

ماجد علي بلطية، عبدالمعز حداد أحمد، عبدالغني إبراهيم عبدالغتي

قسم طب وجراحة العيون، كلية الطب بالقاهرة، جامعة الازهر

E-mail: magedboltia@gmail.com

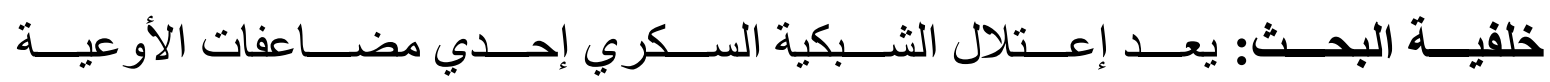
الدموية لداء السكري ومن الأسباب الرئيسية المؤدية إلي العدى.

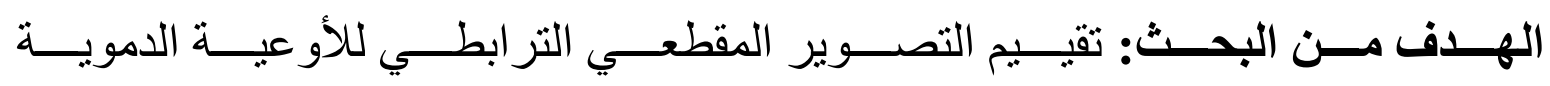

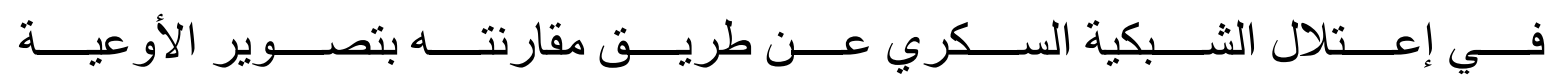
الدموية بالصبغة.

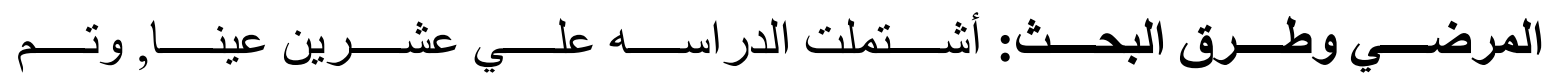

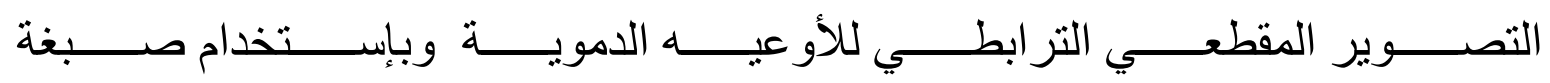

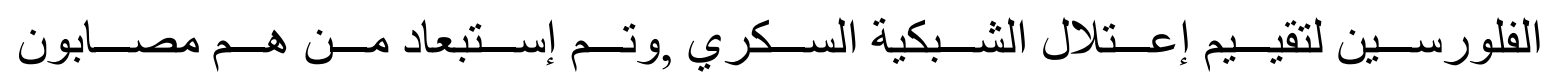
بعتامه في القرنية أو المياه البيضاء أو نزيف بالجسم الزجاجي.

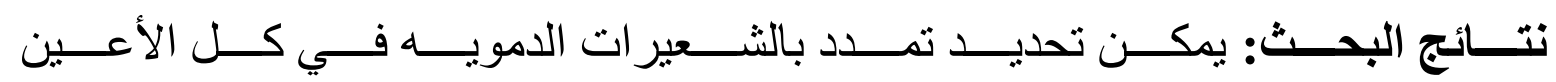

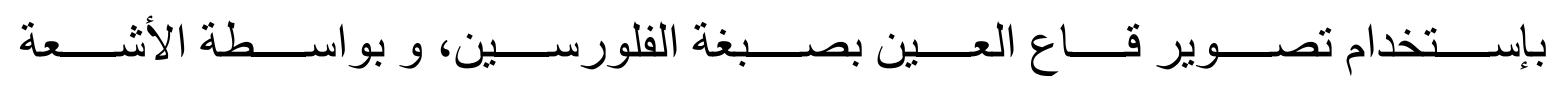

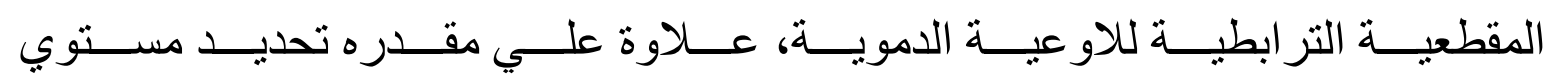

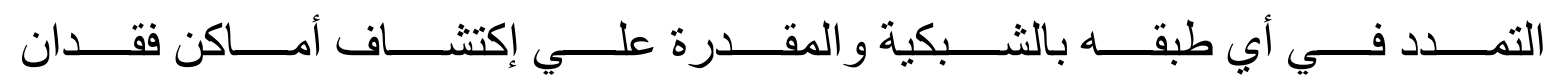

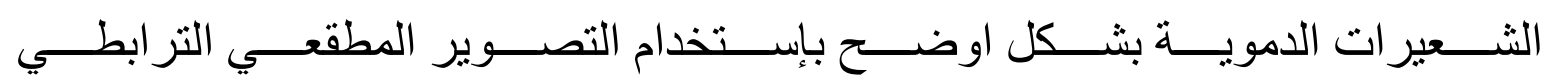

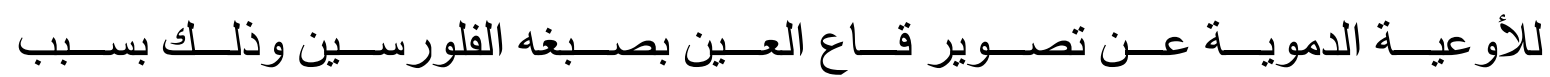

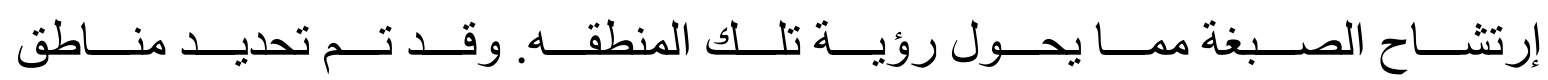

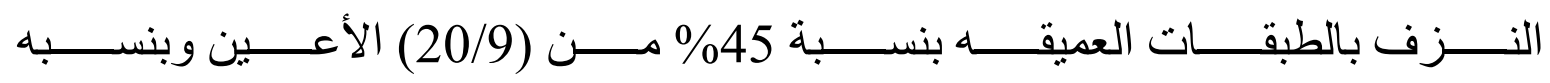

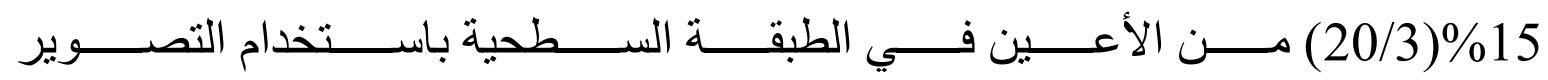
المقطعي الترابطي. 


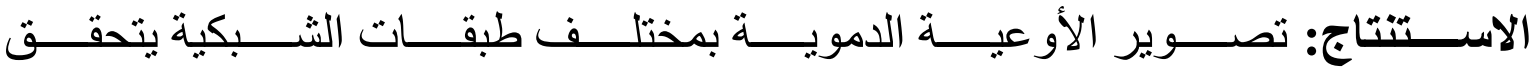

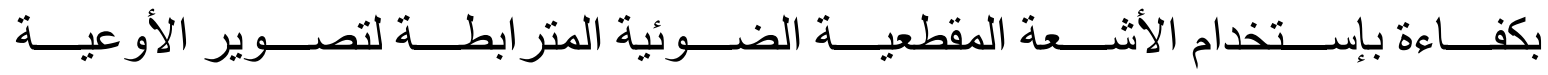

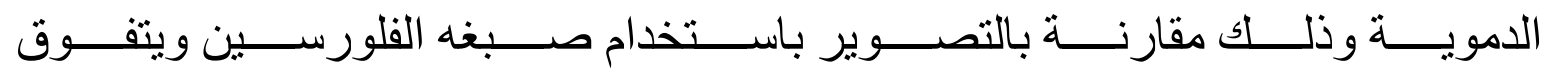

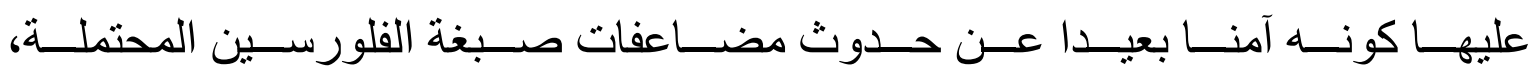

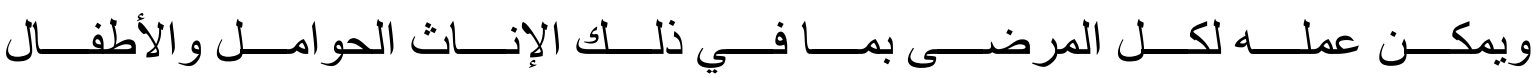

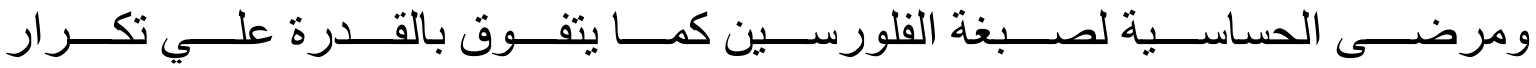

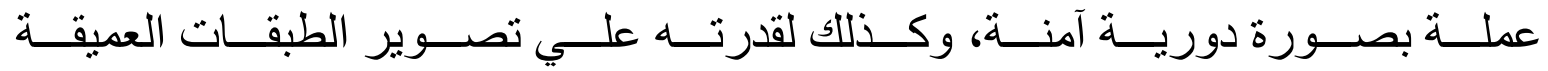

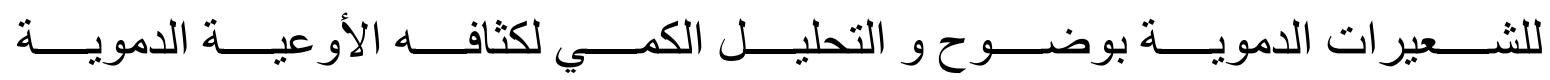
السطحية بمختلف مناطق الثبكية. 